\title{
Improved Bit Allocation Algorithm for Multiview High Efficiency Video Coding
}

\author{
Tao Yan ${ }^{\mathrm{a}, *}$ and In-Ho Ra ${ }^{\mathrm{b}, *}$ \\ ${ }^{a}$ School of Information Engineering, Putian University, Putian, 351100, China \\ ${ }^{b}$ School of Computer, Information and Communication Engineering, Kunsan National University, Gunsan, 54150, South Korea
}

\begin{abstract}
This paper, aiming at the problem of the traditional RD rate control model and inaccurate bit allocation of multi-view high-efficiency video coding (MV-HEVC), proposes a rate control algorithm for MV-HEVC based on the similarity analysis of the views and timedomain complexity of the frame activity. First, the algorithm core uses the similarity analysis of the views to reasonably perform bit allocation among the views. Then, the frame layer and basic unit layer bit allocation and rate control are performed based on the frame rate, target buffer capacity, actual buffer size, and active time domain complexity of the frame. The experimental results show that compared with the fixed bit allocation algorithm applied to views, the proposed method not only effectively controls the bit rate of multiview video coding with a rate control accuracy of over $99 \%$ but also improves the PSNR by $0.38 \mathrm{~dB}$.
\end{abstract}

Keywords: multi-view high efficiency video coding; rate control; bit allocation; similarity analysis

(Submitted on October 22, 2018; Revised on November 21, 2018; Accepted on December 23, 2018)

(C) 2019 Totem Publisher, Inc. All rights reserved.

\section{Introduction}

The most notable features of 3DTV are the interactivity and stereoscopic effect. Users can change the viewpoint position to observe the video content with a three-dimensional (3D) stereoscopic effect according to the actual needs. It has been widely used in advertising, 3D games, 3D movies, exhibitions, and other fields. With the continuous development and application of Internet information technology, it has become difficult for traditional two-dimensional (2D) video technology to meet the visual needs of its users. Therefore, a new generation of 3D video technology, 3DTV, has emerged [1]. As one of the new generations of 3D video coding standards, multi-view high-efficiency video coding (MV-HEVC) has become a new prominent research area worldwide [2], but there is no effective rate control algorithm.

Rate control has typically been one of the most important technologies in video coding standards. Globally, all the video compression standards leave their code rate control, and their applications are limited. Internationally available video compression standards such as MPEG-4 [3], H.264 [4], HEVC (high-efficiency video coding) [5], and MVC (multi-view video coding) [6] have given standard codes. The rate control model and its rate control technology are well established. At present, MV-HEVC [7], one of the latest 3D video coding standards published in the world, has not yet provided an effective rate control algorithm.

At present, the research on MV-HEVC rate control at home and abroad is relatively less than that on the previous rate control technology. Most of the research is focused on rate control such as MVC, HEVC, and H264. Lim et al. proposed an MVC rate control algorithm based on a binomial model [8]. This algorithm uses the geometric relationship between the disparity prediction and motion prediction to divide all the video frames into several different coding type frames but between the various viewpoints. The parallax prediction characteristics have large differences. Park et al. considered the influence of hierarchical B-frames on the temporal coding layer in MVC [9]. The experimental results showed that this algorithm could also maintain efficient coding efficiency, but the average bit rate of the experimental results was dependent on the test sequence. The control error was above $1 \%$, with which it is difficult to meet the needs of practical applications.

\footnotetext{
* Corresponding author.

E-mail address: yantaoshu@aliyun.com
} 
Lei et al. [10] established an MVC rate control algorithm for the R- $\lambda$ model. The experimental results were very satisfactory, but the inter-view correlation was not considered. Vizzotto et al. considered only two viewpoints in the rate control algorithm of stereo video coding [11], and because this algorithm is used in MVC, this type is based on TM5. The accuracy of the target bit number allocation deteriorated, resulting in a very high rate control error and difficulty in bit allocation.

There are numerous domestic and foreign scholars engaged in HEVC-based MVC rate control research. Shao [12] et al. proposed using a fixed allocation ratio to assign code rates between the texture depths, but this method did not achieve optimal coding efficiency for different sequences. Xiao [13] et al. proposed a scalable rate allocation algorithm applied to different bandwidths. Fang et al. [14] proposed an analysis model for estimating the virtual visual distortion in 3D videos and combined the frequency domain time domain analysis method to estimate the virtual visual distortion caused by the distortion of the depth image. The estimation model is accurate but complex.

Pan et al. [15] proposed a depth-based 3D-HEVC rate control algorithm with a fixed color and depth code rate ratio of 4:1, but it could not yield the optimal virtual view quality. Xiao et al. [16] proposed a depth and texture grading rate control algorithm. Wang et al. [17] proposed a 3D-HEVC rate control algorithm based on the binomial R-D model. Because of the direct use of the H.264 medium rate control model, the rate control accuracy was low. In [18], the inter-view R-D model was established by the joint texture and the virtual viewpoint distortion function. However, this method did not utilize the inter-viewpoint correlation, and the code rate control accuracy was lower than the average bit rate error of $1.56 \%$.

All the above algorithms do not fully consider the inter-viewpoint correlation. The focus of MV-HEVC rate control is to perform bit-to-view bit allocation. Different 3D videos have different video characteristics and scene parameters. Typically, different 3D videos require different bit allocations to obtain the best coding efficiency, which requires efficient bit allocation for complex 3D video sequences. For the analysis of the characteristics of a multi-view test sequence, MVC of various types of coded frames and improving the existing MV-HEVC rate control algorithm are considered. The experimental simulation results show that the algorithm has a code rate control error of less than $1 \%$ and improves the coding efficiency. In theory, it has reached the basic requirements of practical applications.

\section{A New Bit Rate Control Strategy and Rate Distortion Theory}

MV-HEVC adopts the traditional R-D rate control model, but the rate control effect is generalized. A rate-based control algorithm based on the $\rho$-domain was proposed by He et al. [19] To introduce the $p$-domain code rate model into MVHEVC, we can assume that $\rho$ and the texture part coding bit rate $R(\rho)$ have the following secondary relationship. It can be written as Equation (1):

$$
R(\rho)=\theta_{1} \cdot(1-\rho)^{2}+\theta_{2} \cdot(1-\rho)+\theta_{3}
$$

Where $\theta_{1}, \theta_{2}, \theta_{3}$ are the one-dimensional regression coefficients, and the initial values are set as $0, R / 2$, and 0 , respectively. After each frame is encoded, it is necessary to refresh in the post-encoding stage. It is shown as Equation (2):

$$
\lim _{\rho \rightarrow 1} R(\rho)=0
$$

Then, we study the following $R-\rho$ model, which can be written as Equation (3):

$$
R(\rho)=\theta_{1} \cdot(1-\rho)^{2}+\theta_{2} \cdot(1-\rho)
$$

Where $\theta_{1}, \theta_{2}$ can be given by the following statistical analysis method: suppose that $x_{1}(\rho)=(1-\rho)^{2}, x_{2}=1-\rho$, and let $\left(x_{11}, x_{12}, R_{1}\right),\left(x_{12}, x_{22}, R_{2}\right), \cdots,\left(x_{1 n}, x_{2 n}, R_{n}\right)$ be the existing $n$ sample values, which can be written as Equation (4):

$$
K=\left(\begin{array}{cc}
x_{11} & x_{21} \\
x_{12} & x_{22} \\
\vdots & \vdots \\
x_{1 n} & x_{2 n}
\end{array}\right), \quad R=\left(\begin{array}{c}
R_{1} \\
R_{2} \\
\vdots \\
R_{n}
\end{array}\right), \quad N=\left(\begin{array}{c}
\theta_{1} \\
\theta_{2}
\end{array}\right)
$$

Using multiple regression techniques, the model parameter $N$ can be calculated as Equation (5): 


$$
N=\left(\begin{array}{l}
\theta_{1} \\
\theta_{2}
\end{array}\right)=\left(K^{T} K\right)^{-1} K^{T} R
$$

Where $K^{T}$ is the transpose matrix of $K$ and $\left(K^{T} K\right)^{-1}$ is the inverse matrix of $K^{T} K$.

\section{3D-HEVC Rate Control Algorithm}

To be compatible with the latest video coding standard HEVC, in this paper, the bit allocation and rate control are proposed based on the HEVC rate control algorithm. The most important algorithm solves the problem of bit allocation between the views. The rate allocation is based on the requirements of MVC to ensure the balance of the video quality between the views. The code rate control algorithm is further improved on the basis of previous research [9], and the rate control is performed for the view layer, frame layer, and macroblock layer, respectively. The key steps of the algorithm are as follows:

\subsection{Viewpoint Ratio Distribution}

In this study, a reasonable allocation of bits to different viewpoints is based on the viewpoint similarity and encoded information. Weight $w_{k}$ is used to indicate the degree of importance of viewpoint $k$. A large $w_{k}$ implies an important viewpoint. The total number of bits allocated to the $K^{\text {th }}$ viewpoint $G O P_{K}$ within each coded $G G O P$ picture group is given by Equation (6):

$$
T_{G O P}\left(n_{k, 0}\right)=T_{G G O P}\left(s n_{i, 0}\right) \cdot w_{k}
$$

The initial value of $w_{k}\left(k=0,1,2, L, N_{\text {view }}-1\right)$ is given by Equation (7):

$$
w_{k}=\frac{\frac{1}{N} \cdot \sum_{j=0, j \neq k}^{N-1} S\left(V_{j}, V_{k}\right)}{\sum_{k=0}^{N-1} \frac{1}{N-1} \cdot \sum_{j=0, j \neq k}^{N-1} S\left(V_{j}, V_{k}\right)}
$$

Where $N$ is the number of encoded viewpoints. This study uses the bilinear similarity measure algorithm, which has been successfully used in the field of image retrieval. This algorithm is superior to the traditional distance measurement method, and there are no restrictions such as distance measurement. $E_{j}$ and $E_{k}$ are the $j$ and $k$ viewpoint feature vectors, respectively, and $T$ is the matrix to be studied in this work. $S\left(V_{j}, V_{k}\right)$ represents the viewpoint $V_{j}, V_{k}$ similarity, which can be written as Equation (8):

$$
S\left(V_{j}, V_{k}\right)=\mathbf{E}_{j}^{T} \mathbf{T} \mathbf{E}_{k}
$$

\subsection{Frame Layer Bit Allocation}

The MV-HEVC frame layer rate control algorithm is more complex than the previous H264 frame layer rate control algorithm. MVC has various different types of coded frames. The algorithm uses the geometric relationship between the disparity prediction and motion prediction to divide all the video frames into several different coding type frames. However, the disparity prediction characteristics between the various viewpoints have large differences, and thus the inter-view prediction relationship or inter-temporal prediction relationship is the same. The coded image may have different coding characteristics. At this time, the target bit number calculated by the same model parameter cannot be used, otherwise a certain deviation will occur. We have previously [9] proposed the frame layer target bit allocation algorithm, given in Equation (9), based on the residual energy of the coded frame.

$$
T(j)=\frac{M A D_{j}}{M A D_{a}} \cdot \frac{\left(T-\sum_{m=1}^{M} C_{m}\right)}{M}+C_{j}
$$


In the above equation, $T$ is the sum of the number of bits consumed for encoding an $M$ frame, $M A D_{a}$ represents the average of all the frames, $M A D_{j}$ represents the $M A D$ at frame $j$, and $C_{j}$ and $C_{m}$ respectively occupy bits of the header information of the $j^{\text {th }}$ frame and $m^{\text {th }}$ frame, respectively.

In the multi-view video code, the target bit of the $j^{\text {th }}$ frame assignment is shown as Equation (10):

$$
T_{r}^{\prime}(j-1)=\left[\frac{M A D_{j-1}}{M A D_{a}} \cdot\left(\frac{T_{G O P}\left(n_{x, 0}\right)}{N(i)}-C_{a}\right)+C_{j-1}\right]
$$

Where $C_{a}$ represents the average value of the bits consumed for encoding the header information of the encoded frame in the current $G O P$.

In general, the intensity of the motion of the video sequence governs the size of the active time domain of each frame, i.e., a large image content scene change requires more bits for encoding. The active time domain of each frame is the image content scene change. Less e encoding implies fewer bits are needed for it. To make the MV-HEVC rate control more precise, the rate control method in formula (10) is further improved, and the current frame target bit is calculated by Equation (11):

$$
T_{r}(j)=T_{r}^{\prime}(j-1) \cdot \frac{\sum_{l=1}^{L} W(l) \cdot 2^{n}}{\sum_{l=1}^{L} \frac{M A D_{l}}{M A D_{a}} \cdot W(l)+\sum_{l=1}^{L} W_{B}(l) \cdot\left(2^{n}-1\right)}+T_{j}
$$

Where $T_{j}$ is the bit consumed by the frame header information of frame $j$ and $n$ represents the current time level. $W(l)$ represents the weight of each frame complexity, and $W_{B}(l)$ represents the weight of $\mathrm{B}$ frame.

\subsection{The Bit Allocation of the Basic Unit Layer}

To be compatible with HEVC, the MVC basic unit layer bit allocation algorithm is similar to HEVC. In the HEVC basic unit layer rate control algorithm, the basic unit layer code rate allocation is relatively simple, i.e., the allocated bits of each frame are evenly distributed to each basic unit layer of the frame. The macroblock is encoded using the same quantization parameter, QP. However, in reality, even the macroblocks in the same basic unit differ significantly in the complexity such as image content, texture, and active time domain. Therefore, to make the MV-HEVC rate control more precise, different quantization values are used according to the complexity of the image content, texture, and active time domain, which are calculated by Equation (12):

$$
T_{k}=\left(T_{\text {total }, k}-T_{\text {head }, k}\right) \times \frac{F D(k)}{\sum_{j=k}^{N} \alpha_{k} \times F D(k)}
$$

In the above formula, $T_{\text {total }, k}$ and $N$ represent the total number of remaining bits and the number of remaining basic units, respectively. $T_{\text {head }, k}$ represents the bit consumed by the $k^{\text {th }}$ macroblock header information, and $F D(k)$ represents the active time domain degree of the $k^{\text {th }}$ coded macroblock, which can be written as Equation (13):

$$
F D(k)=\frac{1}{X \cdot Y} \sum_{x=0}^{X} \sum_{y=0}^{Y}\left|I_{k}(x, y)-I_{k-1}(x, y)\right|
$$

In the formula, $X$ and $Y$ are the number of pixel points and $x$ and $y$ are the macroblocks in the horizontal and vertical directions, respectively. $I_{j}(x, y)$ and $I_{j-1}(x, y)$ are the luminance values of the current macroblock and the previous macroblock, respectively. 


\section{Experimental Results}

To verify the effectiveness of the MV-HEVC rate control algorithm proposed in this paper, the algorithm is compared with reference $[4,6,18]$ and the multi-view rate control algorithm uses the fixed bit allocation. This paper uses two different formats of test sequences, including Vassar, Flamenco2, Exit, NS Ballroom for the VGA format, and PoznanHall2 and GT Fly for the HD format. The resolution of the sequence is $640 \times 480$ pixels and $1920 \times 1088$ pixels. The test platform uses the MV-HEVC system platform provided by JCT-3V [7].

Figure 1 shows the coding efficiency of the five rate control algorithms. Reference [4] is based on the JVT-G102 MVHEVC rate control algorithm. Its coding efficiency is relatively low, and the other four algorithms improve the coding efficiency relative to it to varying degrees. The average PSNR gain is more than $0.6 \mathrm{~dB}$. Moreover, compared with the fixed bit allocation fixed point algorithm between the viewpoints, the other three methods improve the coding efficiency to different degrees, and the PSNR gain is $0.2-1.2 \mathrm{~dB}$. Among them, the multi-view coding rate control algorithm based on the view layer of the R-D code rate model proposed by Roodaki et al. [18] has an average PSNR gain of $0.7 \mathrm{~dB}$ or more. Compared with the literature [18] algorithm, the PSNR of this algorithm is improved, and the PSNR gain is 0.1-0.23 dB. The main reason is that the proposed algorithm not only utilizes the correlation analysis between the viewpoints to reasonably allocate the viewpoint bits but also considers the frame and macroblock active time domain complexities to control the bit rate of the view layer, frame layer, and macroblock layer. The frame allocates relatively numerous bits, and the motion gradual frame allocates a correspondingly small number of bits. Compared with the average allocation of JVTG012, the scheme has a large advantage.

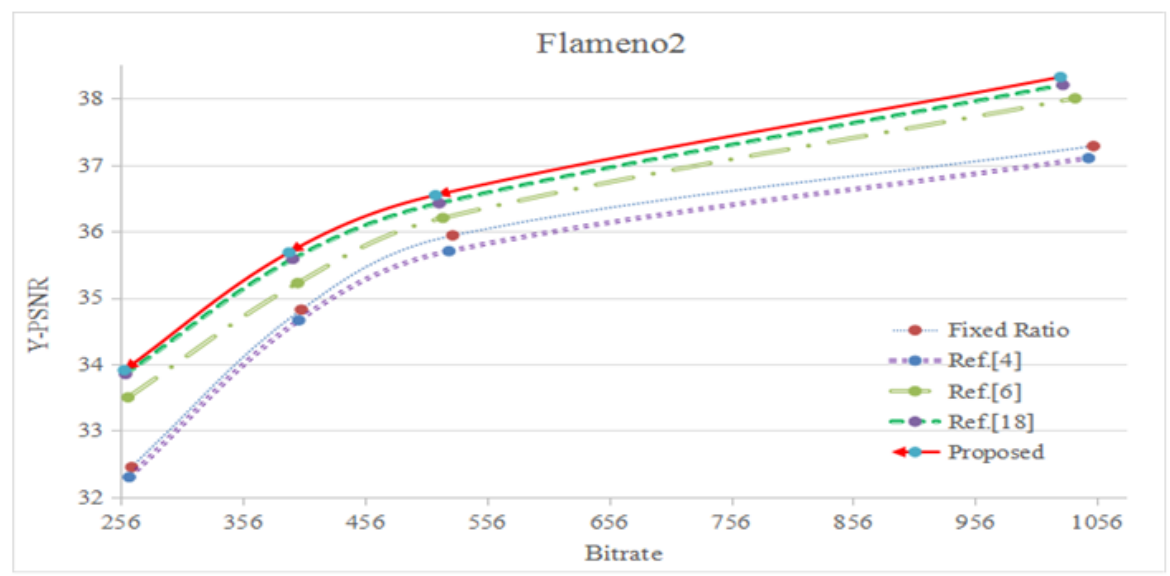

(a) Flameno2 sequence

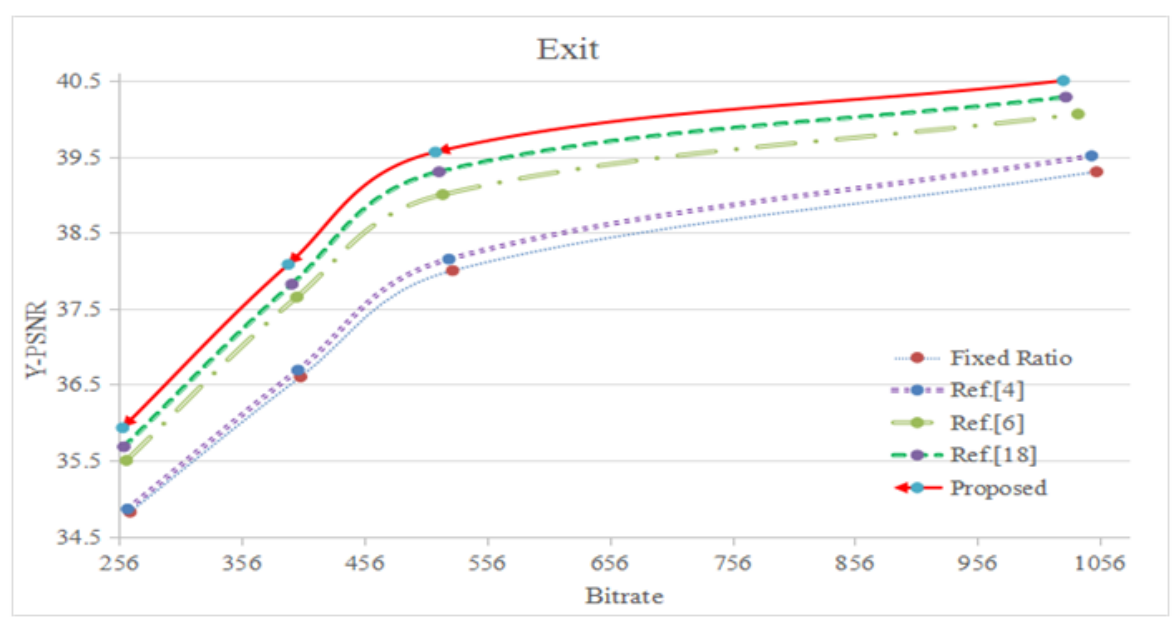

(b) Exit sequence 


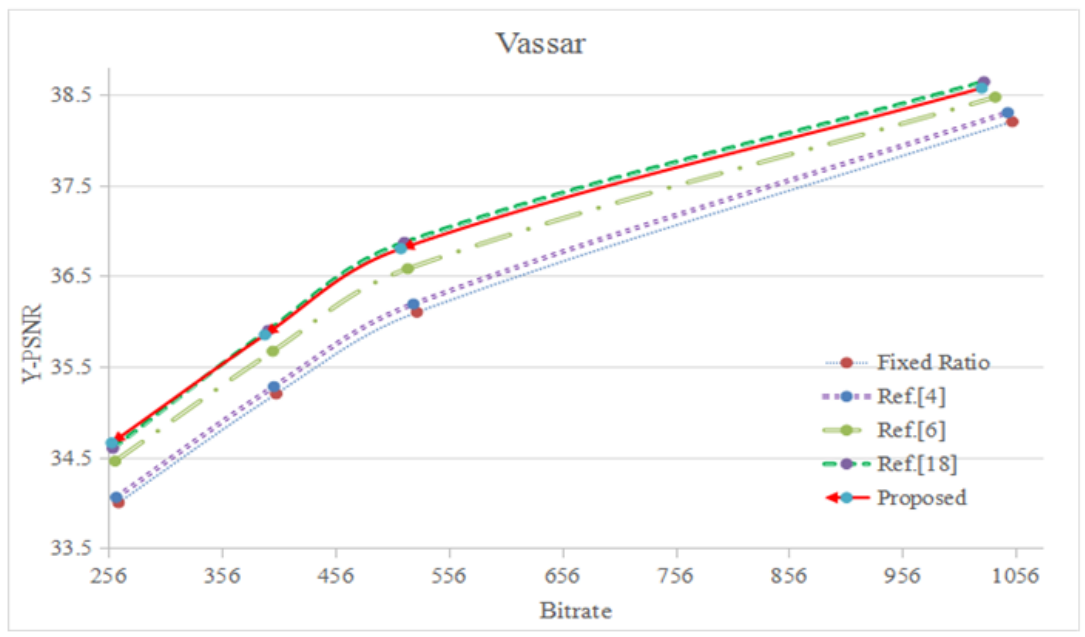

(c) Vassar sequence

Figure 1. PSNR experimental results

Owing to the limited space, this paper only presents the PSNR fluctuation graph of the Vassar sequence. Figure 2 shows that the target bit curve allocated by each frame of the algorithm is consistent with the frame complexity, and the motion striking frame allocates a relatively large number of bits. The motion gradual frame allocation has a correspondingly small number of bits. Compared with literature [6] and [18], this scheme has a significant advantage. It can also be seen from Figure 2 that the proposed rate control scheme increases the frame with a relatively high complexity, improves the PSNR of the motion-sense frame (near the 50th and 77th frames), and reduces the PSNR of the flat frame. Therefore, the fluctuation in the video quality between the frames is significantly improved.

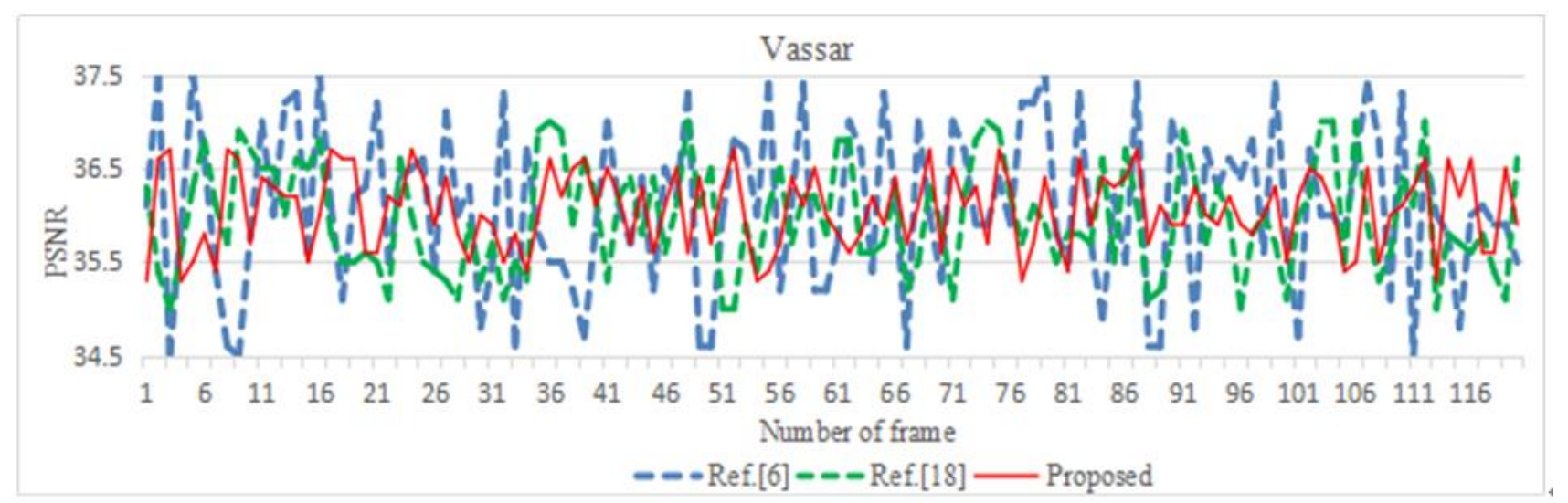

Figure 2. PSNR fluctuation

Table 1 presents the experimental results of the MVC rate control. It can be seen that the code rate control errors in literature [4], [6], and [18] are $2.53 \%, 2.02 \%$, and 1.56\%, respectively, which are relatively large. In this paper, a rate control was proposed. Compared with the other four algorithms, this algorithm has a more accurate bit rate code rate, less code rate deviation, and an average code rate error of less than $1 \%$, which can meet the practical application requirements. The main reason is that a reasonable rate control is performed not only between the viewpoints but also in the frame layer and basic unit layer. Moreover, the number of bits of the motion frame is relatively large, and the motion is moderately allocated. With fewer bits, this solution has a larger advantage than the average allocation of JVT-G012. The PoznanHall2 sequence code rate control error is also relatively large. The main reason is that the correlation between some sequence views is not strong, which results in inaccurate bit allocation between the views and a relatively large final result code rate error.

In this paper, the target bit allocation of the view layer, frame layer, and macroblock layer in MV-HEVC rate control is improved, and a bit allocation scheme based on the view correlation and frame complexity estimation is proposed. A reasonable bit allocation is performed for each viewpoint based on the inter-view correlation analysis to avoid the negative impact of the buffer "liquid level" fluctuation on the frame target bit allocation. The complexity of the current frame is predicted by previous frame MAD, previous frame time domain activity, and current frame time domain activity. The 
proposed frame complexity-based bit allocation overcomes the limitations of the previous research results from target bit allocation.

Table 1. Simulation results

\begin{tabular}{|c|c|c|c|c|c|c|c|c|c|c|c|c|}
\hline \multirow{2}{*}{\multicolumn{2}{|c|}{ Sequence }} & \multirow{3}{*}{$\begin{array}{c}\begin{array}{c}\text { Target Bit rate } \\
(\mathrm{Kbps})\end{array} \\
256 \\
\end{array}$} & \multicolumn{5}{|c|}{ Actual generated bits (kbps) } & \multicolumn{5}{|c|}{ Rate control error $(\%)$} \\
\hline & & & \multirow{2}{*}{$\begin{array}{c}\text { Fixed } \\
\text { Ratio } \\
265 \\
\end{array}$} & \multirow{2}{*}{$\frac{\text { Ref. [4] }}{263}$} & \multirow{2}{*}{$\begin{array}{c}\text { Ref. [6] } \\
262 \\
\end{array}$} & \multirow{2}{*}{$\frac{\text { Ref. [18] }}{260}$} & \multirow{2}{*}{$\begin{array}{c}\text { Proposed } \\
259 \\
\end{array}$} & \multirow{2}{*}{$\begin{array}{r}\begin{array}{r}\text { Fixed } \\
\text { Ratio }\end{array} \\
3.47 \\
\end{array}$} & \multirow{2}{*}{$\frac{\text { Ref. [4] }}{2.66}$} & \multirow{2}{*}{$\begin{array}{c}\text { Ref. [6] } \\
2.27 \\
\end{array}$} & \multirow{2}{*}{$\begin{array}{c}\text { Ref. [18] } \\
1.70 \\
\end{array}$} & \multirow{2}{*}{$\begin{array}{c}\text { Proposed } \\
1.13 \\
\end{array}$} \\
\hline \multirow{15}{*}{ VGA } & & & & & & & & & & & & \\
\hline & \multirow{3}{*}{ Vassar } & 392 & 404 & 402 & 401 & 397 & 394 & 3.16 & 2.55 & 2.18 & 1.25 & 0.53 \\
\hline & & 512 & 528 & 525 & 520 & 517 & 514 & 3.19 & 2.54 & 1.56 & 0.98 & 0.40 \\
\hline & & 1024 & 1053 & 1049 & 1038 & 1028 & 1026 & 2.81 & 2.44 & 1.38 & 0.41 & 0.24 \\
\hline & \multirow{4}{*}{ Flamenco2 } & 256 & 263 & 263 & 263 & 261 & 260 & 2.64 & 2.73 & 2.89 & 1.92 & 1.69 \\
\hline & & 392 & 402 & 402 & 398 & 396 & 393 & 2.52 & 2.55 & 1.60 & 1.00 & 0.27 \\
\hline & & 512 & 527 & 526 & 524 & 519 & 516 & 2.97 & 2.73 & 2.36 & 1.44 & 0.77 \\
\hline & & 1024 & 1059 & 1049 & 1043 & 1038 & 1033 & 3.43 & 2.44 & 1.87 & 1.35 & 0.90 \\
\hline & \multirow{4}{*}{ Exit } & 256 & 263 & 261 & 258 & 257 & 257 & 2.57 & 1.95 & 0.86 & 0.46 & 0.36 \\
\hline & & 392 & 403 & 402 & 402 & 400 & 398 & 2.89 & 2.55 & 2.46 & 1.92 & 1.58 \\
\hline & & 512 & 525 & 523 & 520 & 515 & 515 & 2.59 & 2.15 & 1.65 & 0.66 & 0.56 \\
\hline & & 1024 & 1054 & 1048 & 1043 & 1035 & 1028 & 2.91 & 2.34 & 1.90 & 1.09 & 0.37 \\
\hline & \multirow{3}{*}{ Ballroom } & 392 & 404 & 402 & 401 & 398 & 397 & 3.14 & 2.55 & 2.21 & 1.45 & 1.19 \\
\hline & & 512 & 523 & 523 & 521 & 518 & 517 & 2.20 & 2.15 & 1.83 & 1.17 & 1.00 \\
\hline & & 1024 & 1054 & 1048 & 1045 & 1043 & 1040 & 2.88 & 2.34 & 2.04 & 1.89 & 1.60 \\
\hline \multirow{8}{*}{ HD } & \multirow{4}{*}{$\begin{array}{c}\text { Poznan } \\
\text { Hall2 }\end{array}$} & 1024 & 1060 & 1050 & 1045 & 1048 & 1046 & 3.52 & 2.54 & 2.05 & 2.33 & 2.12 \\
\hline & & 1536 & 1581 & 1578 & 1569 & 1567 & 1562 & 2.91 & 2.73 & 2.15 & 2.01 & 1.68 \\
\hline & & 2048 & 2107 & 2104 & 2089 & 2084 & 2078 & 2.90 & 2.73 & 2.00 & 1.75 & 1.47 \\
\hline & & 4096 & 4209 & 4203 & 4190 & 4164 & 4138 & 2.76 & 2.61 & 2.29 & 1.65 & 1.02 \\
\hline & \multirow{4}{*}{ GT Fly } & 1024 & 1053 & 1049 & 1045 & 1043 & 1037 & 2.79 & 2.44 & 2.05 & 1.82 & 1.31 \\
\hline & & 1536 & 1574 & 1575 & 1568 & 1561 & 1555 & 2.48 & 2.54 & 2.08 & 1.65 & 1.21 \\
\hline & & 2048 & 2105 & 2101 & 2089 & 2084 & 2071 & 2.80 & 2.59 & 2.00 & 1.77 & 1.12 \\
\hline & & 4096 & 4243 & 4219 & 4188 & 4161 & 4146 & 3.58 & 3.00 & 2.25 & 1.59 & 1.23 \\
\hline \multicolumn{2}{|c|}{ Average } & & & & & & & 2.93 & 2.53 & 2.02 & 1.56 & 0.93 \\
\hline
\end{tabular}

In summary, the MV-HEVC rate control scheme was proposed in this paper, and it was found that the previous rate control algorithm could significantly reduce the video quality fluctuations while improving the coding efficiency.

\section{Conclusions}

For MV-HEVC rate control, no in-depth current research has been performed. This paper proposes a new inter-viewpoint rate control algorithm after analyzing the characteristics of insufficient video rate control and multi-view video coding. The algorithm basically involves the entire rate control process, from bit-to-view bit allocation to bit allocation and rate control for each macroblock, thus ensuring the accuracy of the MV-HEVC rate control algorithm. The experimental results show that the proposed bit allocation and rate control algorithm for MV-HEVC can perform effective rate control based on the given coding parameters. In the future, we will further study the correlation between the viewpoints to improve the rate control algorithm more effectively. In addition, this study did not consider the multi-view scene switching and algorithm complexity, which is the focus of future research work.

\section{Acknowledgements}

This work was supported by the Natural Science Foundation of China (No. 61741111, 61302118), Program for New Century Excellent Talents in Fujian ProvinceUniversity, Natural Science Foundation of Jiangxi (No. 20181BAB202011, 20161BAB212031), and National Research Foundation of Korea (NRF) grant funded by the Korean government (MSIP) (No. 2016R1A2B4013002).

\section{References}

1. P. J. Ren, X. Zhang, H. W. Bi, H. Sun, and N. Zheng, "Toward an Efficient Multiview Display Processing Architecture for 3DTV," IEEE Transactions on Circuits and Systems II: Express Briefs, Vol. 64, No. 6, pp. 705-709, 2017

2. J. Y. Lee, J. K. Han, J. G. Kim, and T. Q. Nguyen, "Efficient Inter-View Motion Vector Prediction in Multi-View HEVC," 
IEEE Transactions on Broadcasting, Vol. 64, No. 3, pp. 666-680, 2018

3. F. Pan, Z. G. Li, K. Lim, and G. Feng, "A Study of MPEG-4 Rate Control Scheme and its Implementations," IEEE Transactions on Circuits and System for Video Technology, Vol. 13, No. 5, pp. 440-446, 2003

4. Z. G. Li, F. Pan, K. P. Lim, et al., "Adaptive Basic Unit Layer Rate Control for JVT," in Proceedings of 7th Meeting JVT-G012, pp. 7-14, Pattaya, Thailand: JVT, 2003

5. G. J. Sullivan, J. M. Boyce, Y. Chen, J. R. Ohm, C. A. Segall, and A. Vetro, "Standardized Extensions of High Efficiency Video Coding (HEVC)," IEEE Journal of Selected Topics in Signal Processing, Vol. 7, No. 6, pp. 1001-1016, 2013

6. P. J. Lee and Y. C. Lai, "Vision Perceptual based Rate Control Algorithm for Multi-View Video Coding," IEEE Transactions on Circuits and Systems for Video Technology, Vol. 25, No. 1, pp. 139-152, 2011

7. T. Ikai, K. Kei, and T. Suzuki, "JCT3V-MV-HEVC and 3D-HEVC Conformance Draft 4," in Proceedings of Joint Collaborative Team on 3D Video Coding Extensions (JCT-3V) 14th Meeting: San Diego, ISO/IEC JTC 1/SC 29/WG 11, USA: JCT-3V, pp. 22-26, 2016

8. J. E. Lim, J. Kim, K. Ngan, and K. Sohn, "Advanced Rate Control Technologies for 3D-HDTV," IEEE Transactions on Consumer Electronics, Vol. 49, No. 4, pp. 1-6, 2013

9. T. Yan, P. An, L. Q. Shen, and Z. Y. Zhang, "Bit Allocation and Rate Control Algorithm for MVC," The Imaging Science Journal, Vol. 59, No. 4, pp. 202-210, 2011

10. J. Lei, X. He, H. Yuan, et al., "Region Adaptive R- $\lambda$ Model based Rate Control for Depth Maps Coding," IEEE Transactions on Circuits and Systems for Video Technology, Vol. 28, No. 6, pp. 1390-1405, 2018

11. B. B. Vizzotto, B. Zatt, M. Shafique, et al., "A Model Predictive Controller for Frame-Level Rate Control in Multiview Coding," in Proceedings of the IEEE 12nd International Conference on Multimedia and Expo, pp. 485-490, Melbourne, Australia, 2012

12. F. Shao, G. Y. Jiang, M. Yu, and Q. Dai, "Joint Bit Allocation and Rate Control for Coding Multiview Video Plus Depth based 3D Video," IEEE Transactions on Multimedia, Vol. 15, No. 8, pp. 1843-1854, 2013

13. J. Xiao, M. M. Hannuksela, T. Tillo, and M. Gabbouj, "Scalable Bit Allocation Between Texture and Depth Views for 3-D Video Streaming Over Heterogeneous Networks," IEEE Transactions on Circuits and Systems for Video Technology, Vol. 25, No. 1, pp. 139-152, 2015

14. L. Fang, N. M. Cheung, D. Tian, A. Vetro, H. F. Sun, and O. C. Au, "An Analytical Model for Synthesis Distortion Estimation in 3D Video," IEEE Transactions on Image Processing, Vol. 23, No. 1, pp. 185-199, 2014

15. G. Pan and X. Wei, "Rate-Distortion Optimized Mode Switching for Error-Resilient Multi-View Video Plus Depth based 3-D Video Coding," IEEE Transactions on Multimedia, Vol. 16, No. 7, pp. 1797-1808, 2014

16. J. M. Xiao, M. M. Hannuksela, and T. Tillo, "Scalable Bit Allocation Between Texture and Depth Views for 3-D Video Streaming Over Heterogeneous Networks," IEEE Transactions on Circuits and Systems for Video Technology, Vol. 25, No. 1, pp. 139-152, 2015

17. X. Wang, S. Kwong, H. Yuan, Y. Zhang, and Z. Pan, "View Synthesis Distortion Model based Frame Level Rate Control Optimization for Multiview Depth Video Coding," Signal Processing, Vol. 112, No. 1, pp. 189-198, 2015

18. H. Roodaki, Z. Iravani, M. R. Hashemi, et al., "A View-Level Rate Distortion Model for Multi-View/3D Video", IEEE Transactions on Multimedia, Vol. 18, No. 1, pp. 14-24, 2016

19. Z. H. He and S. K. Mitra, "A Linear Source Model and a Unified Rate Control Algorithm for DCT Video Coding," IEEE Transaction on Circuits for Video Technology, Vol. 12, No. 11, pp. 970-982, 2002

Tao Yan received his Ph.D. in communication and information systems from Shanghai University in 2010. He is currently an associate professor in the School of Information Engineering at Putian University. His major research interests include Multiview high efficiency video coding, rate control, and video codec optimization.

In-Ho Ra received his Ph.D. in computer engineering from Chung-Ang University in 1995. He is currently a professor in the School of Computer, Information, and Communication at Kusan National University. His major research interests include blockchain. 